\title{
Information Rate Loss from Radiation Decoherence
}

\author{
Alfonso Martinez \\ Centrum Wiskunde \& Informatica \\ The Netherlands \\ Email: alfonso.martinez@ieee.org
}

\begin{abstract}
The information rates achievable by using electromagnetic radiation affected by thermal noise and signal decoherence are studied. The classical coherent-state Gaussian model is compared with an alternative photon gas model which represents lack of a shared phase reference between transmitter and receiver. At any frequency, the information rates in the presence of decoherence essentially coincide with those over a Gaussian model when the signal-to-noise ratio is below a threshold. Only above the threshold does decoherence cause a significant loss in information rates; the loss can amount to half of the capacity. The threshold exceeds $40 \mathrm{~dB}$ for radio frequencies and vanishes at optical frequencies.
\end{abstract}

\section{INTRODUCTION}

The consideration of quantum effects in information theory has a rich history, from the pioneering analysis of Gordon [1], [2], through significant contributions by Helstrom [3] and Holevo [4], [5], up to more recent work by Giovannetti et al. [6], [7]. Often, these authors aimed at deriving Shannon's expression for the capacity of a waveform channel with Gaussian noise from basic quantum-mechanical principles. As is well-known, Shannon's formula for the capacity $\mathrm{C}_{\mathrm{Sh}}$ (in bits per Fourier mode) of the Gaussian channel is [8]

$$
\mathrm{C}_{\mathrm{Sh}}\left(E_{s}, \sigma^{2}\right)=\log \left(E_{s}+N_{0}\right)-\log \left(N_{0}\right),
$$

where $E_{s}$ is the average received energy per mode and $N_{0}$ is the one-sided (thermal) noise spectral density.

In Gordon's approach [1], [2], information is sent over coherent states and recovered at the receiver by performing a coherent heterodyne measurement. In this case, noise is additive Gaussian with variance $\left(\varepsilon_{n}+1\right) h \nu$, where $\varepsilon_{n}$ is the average number of thermal photons in the corresponding mode of frequency $\nu$; the average energy $E_{s}$ similarly becomes $E_{s}=\varepsilon_{s} h \nu, \varepsilon_{s}$ being the average number of signal photons. In this case, the capacity $\mathrm{C}_{\mathrm{Het}}$ is given by [1], [9]

$$
\mathrm{C}_{\text {Het }}\left(\varepsilon_{s}, \varepsilon_{n}\right)=\log \left(1+\varepsilon_{s}+\varepsilon_{n}\right)-\log \left(1+\varepsilon_{n}\right) \text {. }
$$

In addition, if one does not impose a restriction on the measurement method, one can use the Holevo-SchumacherWestmoreland (HSW) theorem to compute the largest information rate achievable. When the channel inputs are not entangled and no entangled measurements are allowed one obtains the so-called one-shot capacity. If coherent-i. e. Gaussianstates are used the corresponding one-shot capacity, which we denote by $\mathrm{C}_{\mathrm{HSW}}$, is given by [4], [5], [6]

$$
\mathrm{C}_{\mathrm{HSW}}\left(\varepsilon_{s}, \varepsilon_{n}\right)=g\left(\varepsilon_{s}+\varepsilon_{n}\right)-g\left(\varepsilon_{n}\right),
$$

where $g(t)$ is the entropy of a geometric distribution with mean $t$, given by $g(t)=(1+t) \log (1+t)-t \log t$ (with $0 \log 0=$ $0)$. It has been proved [6] that using entanglement does not increase the capacity in absence of thermal noise, i. e. for $\varepsilon_{n}=0$. For other values of $\varepsilon_{n}$, entanglement might yield a larger capacity, although this formula is conjectured to be the capacity also in that case [7].

When $\varepsilon_{n} \gg 1$, as is the case at radio and microwave frequencies, simple algebra shows that the capacity found by Shannon is very close to the capacity with coherent heterodyne detection and to the one-shot, coherent-state capacity, that is

$$
\mathrm{C}_{\mathrm{Het}}\left(\varepsilon_{s}, \varepsilon_{n}\right) \simeq \mathrm{C}_{\mathrm{HSW}}\left(\varepsilon_{s}, \varepsilon_{n}\right) \simeq \mathrm{C}_{\mathrm{Sh}}\left(E_{s}, k T_{0}\right) .
$$

Any of these equations gives thus the highest information rates achievable in practice when thermal noise is the limiting factor.

Inspired by recent work on reference frames in information theory [10], where Schumacher is quoted as saying that "restrictions on the resources available for communication yield interesting communication theories", we consider the effect of decoherence, by which we mean absence of a shared phase reference between transmitter and receiver, and deal with the information rate loss incurred by such restriction.

In Sec. II we show that decoherence induces a model of radiation as a photon gas, where information is sent by modulating the energy of the Fourier modes of the field; at the receiver, energy is measured. The received signal is the sum of thermal noise, distributed as blackbody radiation at a given temperature and frequency, and a useful signal whose energy distribution is the same as for a coherent state. As with direct detection methods at optical frequencies [9], communication cannot rely on knowledge of the phase of coherent states.

In Sec. III we show that the information rate of the photon gas essentially coincides with Shannon's capacity, with the capacity of heterodyne detection, and with the one-shot coherentstate capacity in Eq. (4) above, provided that the signal-tonoise ratio lies below a threshold; above the threshold, up to half of the capacity may be lost. For a frequency $\nu$ (in Hertz), this threshold is approximately given by $\frac{6 \cdot 10^{12}}{\nu}$ at $290 \mathrm{~K}$, and is thus large for radio and microwave frequencies. Moreover, in the "classical" limit where energy is continuous the capacity of the photon gas coincides with Shannon's capacity $\mathrm{C}_{\mathrm{Sh}}$.

\section{A Model of Radiation as a Photon Gas}

In this section, we present a model of the radiation field detected at the receiver as a photon gas. The model is obtained from the usual quantum analysis by assuming that radiation 
behaves as classical particles, with no quantum interference effects. We hasten to remark that this assumption does not arise naturally from electromagnetic theory. Nevertheless, the model is well-defined and leads to useful insights on the transmission of information by using electromagnetic radiation.

Consider one polarization of the electromagnetic field at an aperture, which we denote by $\tilde{y}(t)$, a complex-valued function representing the positive-frequency components of the received field. Throughout the paper we use a tilde to indicate that the function represents a field amplitude. As is well-known, the field $\tilde{y}(t)$ admits a Fourier decomposition onto frequencies of the form $\nu_{c}+\frac{m}{T}$, lying in a band of width $W$ around a reference frequency $\nu_{c}$; here $T$ is the duration of the observation interval. The $m$-th basis function is then given by $\theta_{m}(t)=\frac{1}{\sqrt{T}} e^{i 2 \pi\left(\nu_{c}+\frac{m}{T}\right) t}$. Further, let the field $\tilde{y}(t)$ represent the superposition of a useful signal $\tilde{x}(t)$ and of additive Gaussian noise $\tilde{z}(t)$, respectively given by

$$
\tilde{x}(t)=\sum_{m} \tilde{x}_{m} \theta_{m}(t), \quad \tilde{z}(t)=\sum_{m} \tilde{z}_{m} \theta_{m}(t) ;
$$

here $\tilde{x}_{m}$ is the field amplitude for the useful signal at mode $m$, set at the transmitter (except for a propagation loss and a phase rotation), and $\tilde{z}_{m}$ are samples of Gaussian noise. Gaussian noise can be naturally associated with thermal radiation at a given temperature $T_{0}$ and frequency $\nu_{m}$.

In a quantum description, the fields $\tilde{y}(t), \tilde{x}(t)$, and $\tilde{z}(t)$ are replaced by operators representing the positive-frequency components of the vector potential; each Fourier mode represents then one degree of freedom of the electromagnetic field. In particular, the received field $\tilde{y}(t)$ is represented by a set of annihilation operators $\hat{y}_{m}$, one for each mode. The superposition of signal and noise is then represented by a completely positive, trace-preserving map [11], which combines the annihilation operators of the electromagnetic field for the useful signal, denoted by $\hat{x}_{m}$, and additive noise, $\hat{z}_{m}$; this map guarantees that the output operators satisfy the bosonic commutation rules. The superposition is given by [12]

$$
\hat{y}_{m}=\sqrt{\eta} e^{i \phi_{x}} \hat{x}_{m}+\sqrt{1-\eta} e^{i \phi_{z}} \hat{z}_{m} .
$$

Note that the sum of the squared modulus of the coefficients multiplying the signal and noise operators is one. The channel maps the two input annihilation operators onto two outputs, the additional output being

$$
-\sqrt{1-\eta} e^{-i \phi_{z}} \hat{x}_{m}+\sqrt{\eta} e^{-i \phi_{x}} \hat{z}_{m} .
$$

We assume that $\eta, \phi_{z}$ and $\phi_{x}$ are independent of the mode index. Note that the channel propagation loss $\eta$ and phase uncertainty are neatly included in the model.

When the phases $\phi_{z}$ and $\phi_{x}$ are known at the receiver, a coherent detection receiver acts on the annihilation operator $\hat{y}_{m}$ [9] and measures a quantity $\tilde{y}_{m}^{\prime}=\sqrt{\eta} \tilde{x}_{m}+\tilde{z}_{m}^{\prime}$, except for an irrelevant phase. Here $\tilde{x}_{m}$ is set at the transmitter and $\tilde{z}_{m}^{\prime}$ is a Gaussian random variable of variance $\left((1-\eta) \varepsilon_{n}+1\right) h \nu$, where $\varepsilon_{n}$ is the average number of thermal photons.
As an alternative, a direct detection receiver reacts to the instantaneous number operator $\hat{y}_{m}^{\dagger} \hat{y}_{m}$, given by

$$
\begin{aligned}
\hat{y}_{m}^{\dagger} \hat{y}_{m}= & \eta \hat{x}_{m}^{\dagger} \hat{x}_{m}+(1-\eta) \hat{z}_{m}^{\dagger} \hat{z}_{m} \\
& +\left(\sqrt{\eta(1-\eta)} e^{i\left(\phi_{x}-\phi_{z}\right)} \hat{x}_{m}^{\dagger} \hat{z}_{m}+\text { c. c. }\right) .
\end{aligned}
$$

Integration of the number operator $\hat{y}_{m}^{\dagger} \hat{y}_{m}$ during the observation interval generates an output, modelled as a random variable $y_{m}$ distributed according to a Laguerre distribution with parameters $\eta \frac{\left|\tilde{x}_{m}\right|^{2}}{h \nu}$ and $(1-\eta) \varepsilon_{n}$ [13]. In the approximation that the energy is continuous, $y_{m}$ follows a non-central chisquare distribution.

Decoherence is equivalent to postulating the removal of the interference term $\hat{x}_{m}^{\dagger} \hat{z}_{m}$ (and its complex conjugate), whose form is that of a quantum interference term, while maintaining the rest of the analysis. The measurement $y_{m}$ is now given by

$$
y_{m}=\int\left(\eta \hat{x}_{m}^{\dagger} \hat{x}_{m}+(1-\eta) \hat{z}_{m}^{\dagger} \hat{z}_{m}\right) d t
$$

namely the sum of the energies of signal and noise. The signal component is modelled as a Poisson random variable, of mean $\eta \frac{\left|\tilde{x}_{m}\right|^{2}}{h \nu}$, where $\tilde{x}_{m}$ is the value fixed at the transmitter, and the additive noise has a geometric distribution [9] of mean $(1-\eta) \varepsilon_{n}$, where $\varepsilon_{n}$ is the average number of thermal photons at the corresponding frequency and temperature. Since $\eta \leq$ 1 , the distributions of signal and noise components remain Poisson and geometric, with the respective means reduced by the corresponding factor, $\eta$ or $1-\eta$ [13].

One can think of this model as a photon gas, where the receiver counts the number of photons in each Fourier mode.

\section{INFORMATION RATES}

In the previous section we introduced a representation of radiation as a photon gas, for a channel model of the form

$$
y_{m}=s_{m}\left(x_{m}\right)+z_{m}, \quad m=1, \ldots, n,
$$

where $y_{m}$ is a measurement on the $m$-th Fourier mode, $x_{m}$ is the $m$-th signal component, a non-negative real number set at the transmitter, $s_{m}$ the useful signal at the output, and $z_{m}$ is the $m$-th sample of additive noise. By construction, signal $s_{m}$ and noise $z_{m}$ are mutually independent; the noise components $z_{m}$ are also independent for different values of $m$.

We further distinguish two variants:

1) For discrete energy, $y_{m}, s_{m}$, and $z_{m}$ are numbers of photons, each of energy $h \nu$. The signal component $s_{m}$ has a Poisson distribution with mean $\eta x_{m}$, where $\eta$ is a propagation loss between transmitter and receiver. In field notation, $x_{m}=\frac{\left|\tilde{x}_{m}\right|^{2}}{h \nu}$. The noise component $z_{m}$ has an geometric distribution with mean $\varepsilon_{n}=(1-\eta)\left(e^{\frac{h \nu}{k T_{0}}}-\right.$ $1)^{-1}$, i. e. thermal radiation at temperature $T_{0}$ attenuated by a factor $(1-\eta)$.

2) For continuous energy, that is $\varepsilon_{s} \gg 1$ and $\varepsilon_{n} \gg 1$, then $y_{m}, s_{m}=\eta x_{m}$ and $z_{m}$ are non-negative real numbers, the energy in the $m$-th mode. The density of the random variable signal energy $h \nu S_{m}$ approaches a delta function, $p_{S \mid X}\left(s_{m} h \nu \mid x_{m} h \nu\right) \rightarrow \delta\left(\left(s_{m}-x_{m}\right) h \nu\right)$. 
Also, $z_{m}$ are samples of exponential noise with mean $E_{n}=(1-\eta) \frac{k T_{0}}{h \nu}$.

In all cases, we impose a constraint on the average received signal energy $E_{s} ; E_{s}$ is related to the average transmitted energy $E_{t}$ as $E_{s}=\eta E_{t}$. We denote by $\varepsilon_{s}$ the average number of received signal photons. We consider only narrowband channels, for which the frequency $\nu$ is constant for all modes.

\section{A. Capacity of the Photon Gas}

In the photon gas model, two sources of noise are present at the output: Poisson noise, arising from the signal itself, and additive noise. Distinct behaviour is to be expected depending on which noise prevails.

In a first approximation, the behaviour is determined by the noise variance. The additive noise variance is given by $\varepsilon_{n}(1+$ $\varepsilon_{n}$ ) (it follows a geometric distribution), whereas the average signal variance is $\varepsilon_{s}$ (as befits a Poisson random variable) [13]. For $\varepsilon_{n} \gg 1$, a region of practical importance, the variances coincide if $\varepsilon_{s}=\varepsilon_{n}^{2}$. We denote this value of $\varepsilon_{s}$ by $\varepsilon_{s}^{*}$. For lower values of $\varepsilon_{s}$, additive noise prevails; at higher signal energies, Poisson noise dominates.

In fact, for any input $p_{X}(x)$ the mutual information satisfies

$$
\begin{aligned}
I(X ; Y) & =H(Y)-H(Y \mid X) \\
& \leq g\left(\varepsilon_{s}+\varepsilon_{n}\right)-H(S(X)+Z \mid X),
\end{aligned}
$$

as the geometric distribution has the highest entropy under the given constraints [8]. Then,

$$
H(S(X)+Z \mid X) \geq H(Z \mid X)=H(Z),
$$

because the entropy of a sum of two independent random variables is at least as large as than the entropy of each of them (Exercise 18 of Chapter 2 of [8]) and $Z$ and $X$ are independent. Therefore,

$$
I(X ; Y) \leq g\left(\varepsilon_{s}+\varepsilon_{n}\right)-g\left(\varepsilon_{n}\right) .
$$

The variables $X, S(X)$, and $Y(S)$ form a Markov chain in this order, $X \rightarrow S(X) \rightarrow Y=S(X)+Z$, so that an application of the data processing inequality [8] yields

$$
I(X ; Y) \leq I(X ; S(X)),
$$

that is the mutual information achievable in the discrete-time Poisson channel; a good upper bound to the capacity of the latter was given in [14].

Hence, the capacity $\mathrm{C}\left(\varepsilon_{s}, \varepsilon_{n}\right)$ of the photon gas model is upper bounded by

$$
\mathrm{C}_{\mathrm{Upp}}\left(\varepsilon_{s}, \varepsilon_{n}\right)=\min \left(\mathrm{C}_{\mathrm{G}}\left(\varepsilon_{s}, \varepsilon_{n}\right), \mathrm{C}_{\mathrm{P}}\left(\varepsilon_{s}\right)\right),
$$

where $C_{G}$ and $C_{P}$ are respectively given by

$$
\begin{aligned}
& \mathrm{C}_{\mathrm{G}}\left(\varepsilon_{s}, \varepsilon_{n}\right)=g\left(\varepsilon_{s}+\varepsilon_{n}\right)-g\left(\varepsilon_{n}\right)=\mathrm{C}_{\mathrm{HSW}}, \\
& \mathrm{C}_{\mathrm{P}}\left(\varepsilon_{s}\right)=\log \left(\left(1+\frac{\sqrt{2 e}-1}{\sqrt{1+2 \varepsilon_{s}}}\right) \frac{\left(\varepsilon_{s}+\frac{1}{2}\right)^{\varepsilon_{s}+\frac{1}{2}}}{\sqrt{e} \varepsilon_{s}^{\varepsilon_{s}}}\right) .
\end{aligned}
$$

In particular, the one-shot capacity of the quantum channel with coherent states, $\mathrm{C}_{\mathrm{HSW}}$, is an upper bound to the capacity of the photon gas model. Moreover, a second bound $\mathrm{C}_{\mathrm{P}}$ may also be active.

Both functions $\mathrm{C}_{\mathrm{G}}$ and $\mathrm{C}_{\mathrm{P}}$ are monotonically increasing functions of $\varepsilon_{s}$. Further, $\mathrm{C}_{\mathrm{P}}$ exceeds $\mathrm{C}_{\mathrm{G}}$ for sufficiently high signal energy. Both bounds thus have a crossing point, whose position we next determine for high signal and noise energy levels, i. e. $\varepsilon_{n} \gg 1$ and $\varepsilon_{s} \gg \varepsilon_{n}$. Simple algebra shows that

$$
\mathrm{C}_{\mathrm{G}}\left(\varepsilon_{s}^{*}, \varepsilon_{n}\right) \simeq \log \left(\frac{\varepsilon_{s}^{*}}{\varepsilon_{n}}\right) \simeq \frac{1}{2} \log \left(\varepsilon_{s}^{*}\right) \simeq \mathrm{C}_{\mathrm{P}}\left(\varepsilon_{s}^{*}\right),
$$

and we obtain again the expression $\varepsilon_{s}^{*}=\varepsilon_{n}^{2}$, previously derived by reasoning in terms of noise variance.

In this classical limit, in the sense of large photon counts, we may use the classical average signal-to-noise ratio SNR, $\mathrm{SNR}=E_{s} / E_{n}$. Further, we assume that $\eta \ll 1$, so that $E_{n}=$ $h \nu \varepsilon_{n}$ is approximately given by $k T_{0}$ as $\varepsilon_{n} \simeq\left(k T_{0}\right) /(h \nu)$. Then, we can define a threshold signal-to-noise ratio $\mathrm{SNR}^{*}$ as

$$
\mathrm{SNR}^{*}=\frac{E_{s}}{E_{n}} \simeq \frac{\varepsilon_{s}^{*} h \nu}{k T_{0}} \simeq \frac{\varepsilon_{n}^{2} h \nu}{k T_{0}} \simeq \frac{k T_{0}}{h \nu} \simeq \frac{6 \cdot 10^{12}}{\nu},
$$

where in the last equation we took $T_{0}=290 \mathrm{~K}$. In decibels, $\mathrm{SNR}^{*}(\mathrm{~dB}) \simeq 37.8-10 \log _{10} \nu(\nu$ in $\mathrm{GHz})$.

The threshold in the upper bounds is mirrored by a similar behaviour for lower bounds. First, we numerically compute a numerical lower bound $\mathrm{C}_{\text {Low }}$, namely the largest of the mutual informations achieved by one of the following two input densities (for $x \geq 0$ ):

$$
\begin{aligned}
& p_{X}(x)=\frac{\varepsilon_{s}}{\left(\varepsilon_{s}+\varepsilon_{n}\right)^{2}} e^{-\frac{x}{\varepsilon_{s}+\varepsilon_{n}}}+\frac{\varepsilon_{n}}{\varepsilon_{s}+\varepsilon_{s}} \delta(x) \\
& p_{X}(x)=\frac{\left(1+2 \varepsilon_{s}\right)^{1 / 2}}{\sqrt{2 e}-1+\left(1+2 \varepsilon_{s}\right)^{1 / 2}} \frac{1}{\sqrt{2 \pi x \varepsilon_{s}}} e^{-\frac{x}{2 \varepsilon_{s}}} \\
& \quad+\frac{\sqrt{2 e}-1}{\sqrt{2 e}-1+\left(1+2 \varepsilon_{s}\right)^{1 / 2}} \delta(x) .
\end{aligned}
$$

The first density is also the optimum input distribution for the additive exponential noise channel, as determined by Verdú [15]. As he found for the continuous-energy case, it is easy to show that the channel output $Y$ follows a geometric output distribution with mean $\varepsilon_{s}+\varepsilon_{n}$ when the input $X$ is distributed according to this density and additive geometric noise $Z$ is added. As for the second density, it was used in [14] to derive an upper bound to capacity of a discrete-time Poisson channel, specifically the formula for $\mathrm{C}_{\mathrm{P}}$.

The threshold can be seen in Fig. 1, which depicts the upper and lower bounds to the capacity as a function of the input number of quanta $\varepsilon_{s}$ and for several values of $\varepsilon_{n}, 1,10^{3}$, and $10^{6}$ thermal photons. The loss in the photon gas model is negligible when, say, $\varepsilon_{s}<\frac{1}{10} \varepsilon_{s}^{*}$. On the other hand, above the energy level $10 \varepsilon_{s}^{*}$, the upper bound $\mathrm{C}_{\mathrm{P}}$ becomes dominant, and eventually half of the achievable information rate is lost compared to Shannon's capacity for coherent models. As the upper and lower bounds are very close, we conclude that the capacity is closely given by the upper bound in Eq. (16). Around the threshold a small gap of about 1 bit between the upper and lower bounds is visible. 


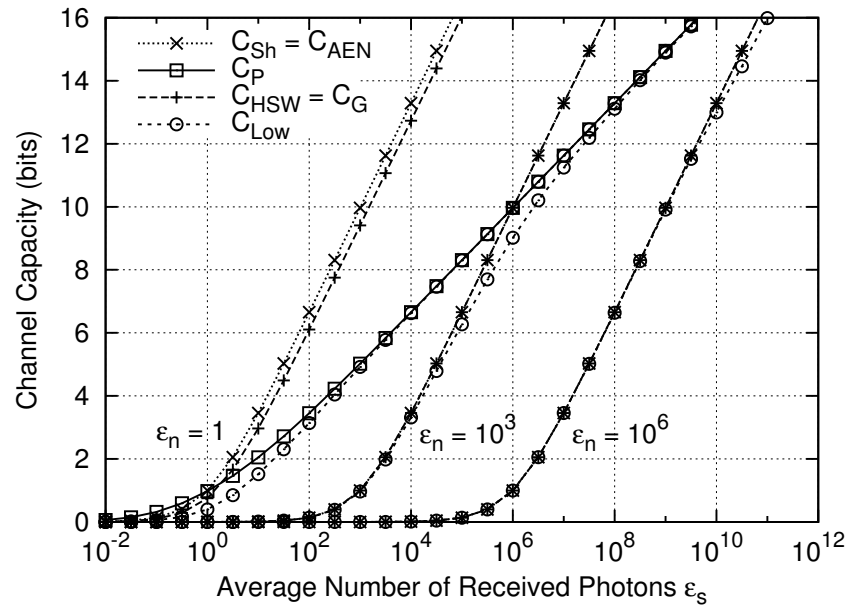

Fig. 1: Bounds to the capacity for several values of $\varepsilon_{n}$.

Simple algebra shows that $\mathrm{C}_{\mathrm{G}}\left(\varepsilon_{s}, \varepsilon_{n}\right)$ is bounded by

$$
\log \left(1+\frac{\varepsilon_{s}}{\varepsilon_{n}+1}\right)<\mathrm{C}_{\mathrm{G}}\left(\varepsilon_{s}, \varepsilon_{n}\right)<\log \left(1+\frac{\varepsilon_{s}}{\varepsilon_{n}}\right)
$$

for finite values of $\varepsilon_{s}$. The upper bound $\mathrm{C}_{\mathrm{G}}\left(\varepsilon_{s}, \varepsilon_{n}\right)$, which is also the one-shot capacity of the quantum model with coherent states $\mathrm{C}_{\mathrm{HSW}}$, is strictly upper bounded by Shannon's classical capacity and lower bounded by the capacity of heterodyne detection. Moreover, the gap between the various capacities vanishes as $\varepsilon_{s}$ and $\varepsilon_{n}$ go to infinity. Shannon's classical capacity, Eq. (1), is also depicted in Fig. 1. For $\varepsilon_{n}=10^{3}$ and $10^{6}, \mathrm{C}_{\mathrm{Sh}}$ is indeed indistinguishable from $\mathrm{C}_{\mathrm{G}}$. However, for $\varepsilon_{n}=1$, Shannon's capacity exceeds the result derived from quantum theory by an amount of about 0.56 bits; we should note here that this low value of $\varepsilon_{n}$ is beyond the classical context where Shannon derived his capacity formula.

In addition, the plots also depict $\mathrm{C}_{\mathrm{Exp}}$, a closed-form lower bound to the capacity by using the density in Eq. (20).

Figure 2 depicts the information rate loss between the conjectured quantum channel capacity $\mathrm{C}_{\mathrm{HSW}}$ and the various upper and lower bounds. The gap is rather small for energies sufficiently below the threshold, progressively approaching half of the capacity as the input energy grows. For $\mathrm{C}_{\mathrm{Exp}}$ the looseness at low $\varepsilon_{s}$ is due to a pessimistic estimate of the conditional output entropy $H(Y \mid X)$, which is smaller than the Gaussian approximation we have used. At high $\varepsilon_{s}$ the tiny gap between $C_{\text {Exp }}$ and $C_{P}$ is caused by the non-optimal input distribution; a closed-form expression derived from Eq. (21) would likely close this gap. The capacity of the photon gas essentially coincides with that of the coherent-state models, even though the phase of the coherent state is not used to transmit information.

At radio and microwave frequencies and for not extremely high signal-to-noise ratios, there are thus four models which give essentially the same channel capacity. A connection, worthwhile mentioning, can be made with non-coherent communications in Gaussian channels [16], where one of the two

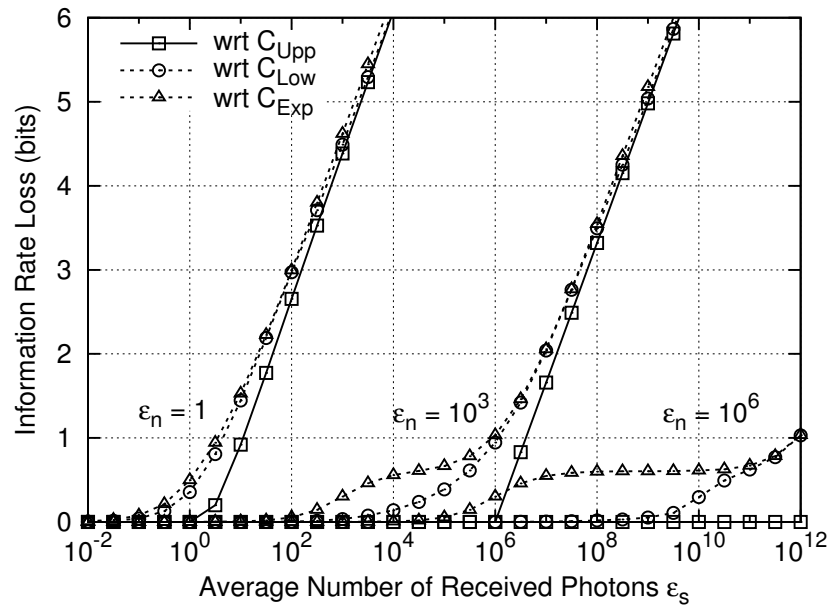

Fig. 2: Bounds to the capacity for several values of $\varepsilon_{n}$.

signal quadratures is not used, and a change in slope of the capacity function from $\log \mathrm{SNR}$ to $\frac{1}{2} \log \mathrm{SNR}$ (for high SNR) occurs. A similar limitation arises in phase-noise limited channels [17]. As the threshold $\varepsilon_{s}^{*}$ is close to the point where existing digital communication systems using electromagnetic radiation suffer from the effects of phase noise, it would be interesting to verify which of the models, coherent detection or the photon gas, defines most accurately the effective channel capacity. Regarding this issue, note that the cost in information rates of maintaining the phase coherence between transmitter and receiver are usually ignored.

We next consider optical frequencies, for which $\varepsilon_{n}$ vanishes. In this case, it is well-known that optical heterodyne coherent detection is close to optimal for large signal energies, in the sense that almost $100 \%$ of the classical capacity $\mathrm{C}_{\mathrm{HSW}}$ can be achieved - the absolute difference between the two capacities quickly approaches 1.44 bits, which becomes negligible if the capacity is large enough.

Moreover, the capacity with optical direct detection, which corresponds to that of the photon gas, is upper bounded by $C_{P}$, which asymptotically grows as $\frac{1}{2} \log \varepsilon_{s}$, and lower bounded by the mutual information achieved by the density in Eq. (21), or by $\mathrm{C}_{\mathrm{Exp}}$. In either case, direct detection and therefore the capacity of the photon gas, is lower by about a factor $\frac{1}{2}$ than the capacity of the coherent-state models.

At low values of the signal energy, as discussed by Gordon [1], the capacity of homodyne coherent detection, $\mathrm{C}_{\mathrm{Hom}}$, exceeds that of heterodyne detection by a factor of 2 . This follows from the formula for $\mathrm{C}_{\mathrm{Hom}}$ [1],

$$
\mathrm{C}_{\text {Hom }}\left(\varepsilon_{s}, \varepsilon_{n}\right)=\frac{1}{2} \log \left(1+\frac{4 \varepsilon_{s}}{2 \varepsilon_{n}+1}\right) .
$$

Further, binary flash signalling, where one symbol is placed at 0 with probability $p$ and another at $1 /(1-p)$ with probability $(1-p)$, achieves a higher mutual information [1]. This is verified in Fig. 3, which depicts the capacity as function of $\varepsilon_{s}$ of flash signalling for several values of $p$, together with the capacities for coherent detection and the conjectured quantum 
capacity $\mathrm{C}_{\mathrm{HSw}}$. The envelope of the capacities with flash signalling is close to the upper bound $\mathrm{C}_{\mathrm{P}}$, which again proves a good estimate of the capacity of the photon gas.

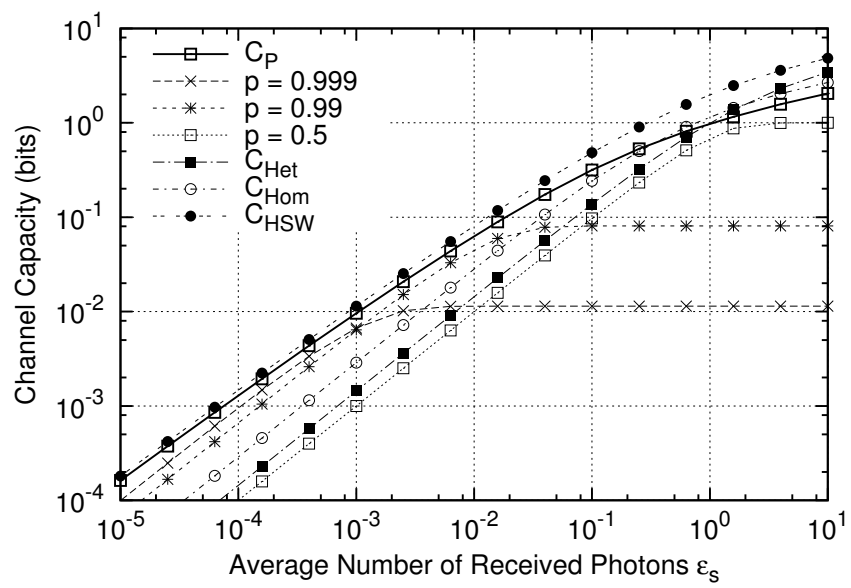

Fig. 3: Discrete-time Poisson channel capacity for flash signalling.

\section{B. Capacity with the Continuous Energy Model}

Under the approximation that the energy is continuous, we previously saw that Poisson noise vanishes and the geometric distribution turns into an exponential density. The capacity of this channel was studied by Verdú [15], who derived the somewhat surprising result

$$
\mathrm{C}_{\mathrm{AEN}}\left(E_{s}, E_{n}\right)=\log \left(E_{s}+E_{n}\right)-\log \left(E_{n}\right) .
$$

Shannon's capacity is thus achieved even though the quadrature components of the field are not explicitly used. On the other hand, this was to be expected since this model is a good description of the regime where $\mathrm{C}_{\mathrm{G}}$ accurately gives the capacity, and the formula here follows from $\mathrm{C}_{\mathrm{G}}$ as $\varepsilon_{n} \rightarrow \infty$.

\section{CONCLUSIONS}

In this paper, we have studied the transmission of information via electromagnetic radiation modelled as a photon gas, that is as an ensemble of photons over a set of Fourier modes. We have seen that the photon gas model need not incur in a significant information rate loss even though the quadrature components of the field are not used separately. In particular, at radio and microwave frequencies, the one-shot capacity of the quantum channel with coherent states, the capacity with heterodyne coherent detection, and the capacity of the photon gas all essentially coincide with Shannon's formula.

We have seen that the entropy of the received signal is determined by that of thermal radiation if the signal energy is below a threshold. Below this threshold, the photon gas model incurs in no information loss; above it, up to half of the channel capacity is lost. The capacity of the photon gas model thus deviates from that of coherent detection at sufficiently high signal-to-noise ratios. For a temperature of $290 \mathrm{~K}$, this threshold signal-to-noise ratio is $\frac{6 \cdot 10^{12}}{\nu}$, well above the operation of most existing communication systems at microwave frequencies. Above the threshold, such as for higher frequencies, the entropy is determined by the noise in the signal itself, a form of shot noise or Poisson noise.

Previous studies of direct detection [16] have shown a nonnegligible capacity penalty. We relate this discrepancy to a different way of accounting for the energy of a mixture of thermal and coherent radiation. In these studies the receiver does not purely detect the sum of the signal and noise energies, but an interference term between signal and noise is present. This term has mean zero but non-zero variance; this variance is the source of the penalty in information rate. In our model, this quantum interference term is made to vanish.

Finally, we mention that the photon gas model is somewhat close to a representation of classical matter as a set of particles, and that the results presented in this paper may be of help in exploring the quantum-classical border for radiation [18].

\section{REFERENCES}

[1] J. P. Gordon, "Quantum effects in communication systems," Proc. IRE, vol. 50, no. 9, pp. 1898-1908, September 1962.

[2] —_, "Noise at optical frequencies; information theory," in Proceedings of the International School of Physics "Enrico Fermi", Course XXXI. London (UK): Academic Press, 1964, pp. 156-181.

[3] C. W. Helstrom, Quantum Detection and Estimation Theory. Academic Press, 1976.

[4] A. S. Holevo, M. Sohma, and O. Hirota, "Capacity of quantum Gaussian channels," Phys. Rev. A, vol. 59, no. 3, pp. 1820-1828, March 1999.

[5] A. S. Holevo and R. F. Werner, "Evaluating capacities of bosonic Gaussian channels," Phys. Rev. A, vol. 63, no. 3, pp. 032 312-1/14, 2001.

[6] V. Giovannetti, S. Guha, S. Lloyd, L. Maccone, J. H. Shapiro, and H. P. Yuen, "Classical capacity of the lossy bosonic channel: The exact solution," Phys. Rev. Lett., vol. 92, no. 2, pp. 027 902-1/4, January 2004.

[7] V. Giovannetti, S. Guha, S. Lloyd, L. Maccone, J. H. Shapiro, and B. J. Yen, "Classical capacity of free-space optical communication," Quant. Inf. Comp., vol. 4, no. 6-7, pp. 489-499, 2004.

[8] T. M. Cover and J. A. Thomas, Elements of Information Theory, ser Wiley Series in Telecommunications. John Wiley and Sons, 1991.

[9] C. M. Caves and P. D. Drummond, "Quantum limits on bosonic communication rates," Rev. Mod. Phys., vol. 66, no. 2, pp. 481-537, April 1994.

[10] S. D. Bartlett, T. Rudolph, and R. W. Spekkens, "Reference frames, superselection rules, and quantum information," Rev. Mod. Phys., vol. 79, no. 2, pp. 555-609, April-June 2007.

[11] M. A. Nielsen and I. L. Chuang, Quantum Computation and Quantum Information. Cambridge (UK): Cambridge Univ. Press, 2000.

[12] R. Loudon, The quantum theory of light, 3rd ed. Oxford (UK): Oxford University Press, 2000.

[13] S. Karp, E. L. O'Neill, and R. M. Gagliardi, "Communication theory for the free-space optical channel," Proc. IEEE, vol. 58, no. 10, pp. 1611-1626, October 1970.

[14] A. Martinez, "Spectral efficiency of optical direct detection," J. Opt. Soc. Am. B, vol. 24, no. 4, pp. 739-749, April 2007.

[15] S. Verdú, "The exponential distribution in information theory," Prob. Per. Inf., vol. 32, no. 1, pp. 86-95, Jan-Mar 1996.

[16] M. Katz and S. Shamai (Shitz), "On the capacity-achieving distribution of the discrete-time noncoherent and partially coherent AWGN channels," IEEE Trans. Inf. Theory, vol. 50, no. 10, pp. 2257-2270, October 2004.

[17] A. Lapidoth, "On phase noise channels at high SNR," in Proc. IEEE Inf. Theory Workshop, Bangalore (India), 2002, pp. 1-4.

[18] W. H. Zurek, "Decoherence and the transition from quantum to classical - revisited," Phys. Today, vol. 44, pp. 36-44, 1991. 